\title{
Formación docente en Chile: percepciones de profesores del sistema escolar y docentes universitarios $^{1}$
}

\author{
Teacher training in Chile: perceptions of teachers in the \\ school system and university teachers
}

Recibido: 12 de diciembre de 2014 - Revisado: 19 de marzo de 2015 - Aceptado: 17 de marzo de 2015

\author{
Claudio H. Díaz Larenas² - María Inés Solar Rodríguez ${ }^{3}$ \\ Valentina Soto Hernández ${ }^{4}$ - Marianela Conejeros del Solar ${ }^{5}$
}

\section{Resumen}

Este artículo presenta los resultados de una investigación sobre percepciones de la formación docente. El diseño del estudio es transeccional y descriptivo, para el cual se elaboró una encuesta ad hoc aplicada a dos grupos: profesores del sistema escolar y docentes universitarios, de la región del Biobío en Chile. Su objetivo es analizar la percepción de estos grupos respecto a seis temáticas: evaluación, aprendizaje, innovación, investigación, perfeccionamiento y relación universidad/ escuela/comunidad. Los principales resultados dan cuenta de una alta valoración de las prácticas evaluativas, la introducción de innovaciones en el aula y el perfeccionamiento. La investigación es realizada tanto por docentes universitarios como por profesores del sistema escolar, aunque en frecuencias distintas. Las relaciones universidad/escuela/comunidad se consideran relevantes, aunque no se encuentran consolidadas. Finalmente, se plantean algunas reflexiones que vinculan las temáticas analizadas en función del mejoramiento de la formación docente.

\section{Palabras clave}

Formación de profesores, profesores, aprendizaje, evaluación, innovación, investigación.

\begin{abstract}
This article presents the results of research on perceptions of teacher training. The study design is transactional and descriptive, for which an ad hoc survey of two groups was developed: the school sysrtem teachers and academics in the region of Bio Bio in Chile. Its aim is to analyze the perception of these groups on six themes: evaluation, learning, innovation, research, development and relationship university / school / community. The main results show a high evaluation of assessment practices, introducing innovations in the classroom and refinement. The reasearch is carried out by both teachers and university profressors in the school system, although at different frequencies. The university / school / community relations are considered relevant but are not consolidarted. Finally some thoughts arise that link the themes discussed in terms of improving teacher education.
\end{abstract}

\section{Keywords}

Teacher training, professors, learning, assessment, innovation, research.
${ }^{1}$ Artículo del proyecto "Acciones integradas para el fortalecimiento científico e institucional en triangulación interna" que forma parte de la Red de Investigación e Innovación Educativa para la mejora de la Educación. Patrocinado por la Agencia Española de Cooperación Internacional para el Desarrollo (AECID), España y liderado por la Universidad de Oviedo, Oviedo, España; con la participación de la Pontificia Universidad Católica de Valparaíso, Valparaíso, Chile; Universidad de Concepción, Concepción, Chile; Universidad Austral de Chile, Valdivia, Chile y Universidad de Talca, Talca, Chile. También se inserta en el contexto del proyecto del Fondo Nacional de Desarrollo Científico y Tecnológico (Fondecyt) "Investigación del conocimiento profesional, las creencias implícitas y el desempeño en aula de estudiantes de Pedagogía en Inglés como estrategia de generación de indicadores de monitoreo de su proceso formativo"

${ }^{2}$ Doctor en Educación. Dirección de Docencia, Facultad de Educación. Universidad de Concepción, Concepción, Chile.

Correo electrónico:

claudiodiaz@udec.cl

${ }^{3}$ Doctora en Filosofía y Ciencias de la Educación. Profesora emérita de la Universidad de Concepción, Concepción, Chile. Asesora académica. Dirección de Docencia, Universidad de Concepción.

Correo electrónico: marsolar@udec.cl

${ }^{4}$ Socióloga y magíster en Sociología. Asesora de estudios. Dirección de Docencia, Universidad de Concepción, Concepción, Chile.

Correo electrónico:

sotohernandez.valentina@gmail.com ${ }^{5}$ Magíster en Educación. Académica de la Dirección de Educación Continua de la Universidad de Concepción, Concepción, Chile.

Correo electrónico:

conejerosmarianela@gmail.com

Para citar este artículo: Díaz, C., Solar, M., Soto, V., \& Conejeros Del, M. (2015). Formación docente en Chile: percepciones de profesores del sistema escolar y docentes universitarios. Revista Civilizar Ciencias Sociales y Humanas, 15(28), 229-246. 


\section{Introducción}

En la actualidad existe en las instituciones de educación superior preocupación por responder desde la academia, a las demandas del sector productivo, a los requerimientos de los empleadores y a la formación de profesionales capaces y creativos. No solo contestar con eficiencia a los problemas de la práctica profesional, sino también lograr un desempeño ético y socialmente responsable (Centro Interuniversitario de Desarrollo, Cinda, 2009). La Organización para la Cooperación y el Desarrollo Económicos (OCDE) (2013) expresa que las políticas públicas que se ejecutan en diferentes países, están crecientemente centradas en la calidad de los desempeños en su conjunto. Por esto, es indispensable un proceso evaluativo con el objeto de mejorar y entregar información a la sociedad, a los que elaboran las políticas públicas, a las escuelas y a las familias (p. 3).

Por lo común, las universidades han sido lentas para abordar las realidades emergentes que se vinculan a la definición de resultados de aprendizaje de acuerdo con desempeños medibles, acreditables y diversos en opciones de enseñanza (Cárdenas, Rodríguez, \& Torres, 2000; Torre De la, 2004). Incertidumbre, complejidad e impredecibilidad, es lo que caracteriza cada vez más, al mundo actual. Gran parte de este desafío involucra a las universidades que deben elaborar las implicancias operativas, sociocognitivas, afectivas y pedagógicas para responder a las demandas de la sociedad global y a los nuevos escenarios de aprendizaje (Solar, 2013).

Los inicios del siglo XXI generaron cambios en la academia que tienen en cuenta la sociedad del conocimiento y los nuevos requerimientos para el ejercicio profesional (Frabboni, 2001; Hashweh, 2005). En este sentido, la Organización de las Naciones Unidas para la Educación, la Ciencia y la Cultura (Unesco, por su acrónimo en inglés) (1998), destaca que "la educación superior está siendo desafiada a considerar sus objetivos fundamentales, a encontrar un equilibrio entre la búsqueda de conocimientos por sí mismo y el servicio directo a la sociedad, a fomentar capacidades genéricas e impartir conocimientos especializados" (p. 12).

El sistema educativo chileno ha implementado acciones para responder a estos retos. Sin embargo, las deficiencias persisten en distintos niveles. Por ejemplo, el informe Pisa ${ }^{1}$ da cuenta de las variables más significativas para la comprensión de esta situación, tales como las condiciones de descentralización del sistema, deficiente formación del profesorado y precarias condiciones de vida de muchos estudiantes que experimentan la marginación cultural, social y económica; lo que dificulta los procesos de aprendizaje.

Todo ello exige actuar en un proceso de mejoramiento de la formación de docentes, en particular, en aspectos como la investigación y los modelos de intervención, innovación educativa y gestión, tanto en el ámbito universitario como extrauniversitario (Joyce, Marsha, \& Calhoun, 2002; Kansanen, Tirri, Meri, Krokfors, Husu, \& Jyrhämä, 2000). Este proceso se sustenta en la actividad investigadora como potencial motor de la revolución educativa que se necesita en el sistema educativo chileno, especialmente en aquella parte que atiende a la mayoría de la población cuyos establecimientos escolares pertenecen al sector público o al semipúblico, por ser ambos los más precarios en recursos, calidad de sus docentes y gestión. Esta posibilidad de mejora solo pueden realizarla las universidades como agentes críticos, indagadores, reflexivos e interventores desde su misión (Gonzáles, Río, \& Rosales, 2001; Libedinsky, 2001; Médina \& Salvador, 2002; Menin, 2001).

Las universidades en general, y las latinoamericanas en particular, urgen de incentivos y apoyos externos que aporten recursos para la formación, investigación y gestión (Monereo, Castillo, Clariana, Palma, \& Pérez, 2004; Muchmore, 
2004; Neary, 2002; Roberts, 2002). Si bien los recursos son de naturaleza muy diversa (material, humana y económica), los más importantes sin duda, son los humanos. Cisternas (2013) indica que los estudios que se relacionan con la práctica de la investigación, evidencian la importancia que tiene el contexto y la contingencia en el proceso de decidir qué investigar. $\mathrm{La}$ autora se pregunta qué procesos y dimensiones intervienen en la definición de los objetos de estudio, que abordan las investigaciones acerca de la formación docente (p. 117).

Son escasos los estudios que afrontan los interrogantes en torno a la producción de conocimiento en el ámbito educativo (Sandín, 2003; Sanjurjo, 2002; Scharle \& Szabó, 2000). No obstante, a partir de la investigación se pueden reorientar las prácticas docentes y redefinir los planes de estudio en la coherencia teoríapráctica, hacia la formación de competencias en los docentes. Es menester analizar y diagnosticar las dificultades para atender los retos planteados internamente -formar docentes- y externamente -dar respuesta a las necesidades de la sociedad-.

El establecimiento de criterios para guiar el desempeño profesional docente comienza en Chile con el Marco para la buena enseñanza (2003), documento elaborado conjuntamente por el Ministerio de Educación, el Colegio de Profesores y la Asociación Chilena de Municipalidades, a partir del cual se delimitaron los siguientes dominios:

- Dominio A: preparación de la enseñanza.

- Dominio B: creación de un ambiente propicio de aprendizaje.

- Dominio C: enseñanza para el aprendizaje de todos los estudiantes.

- Dominio D: responsabilidades profesionales (Ministerio de Educación, 2013).

En el año 2004, un informe de la OCDE sobre las políticas y resultados educacionales, señaló en forma explícita que las políticas debían abordar sistemáticamente el problema de la preparación de los docentes, con especial foco en los conocimientos disciplinarios, puesto que, en caso contrario, no existirían cambios en los resultados de aprendizaje de los estudiantes chilenos.

Las conclusiones de este estudio destacan que las universidades requieren contar con información sistemática y fidedigna de las necesidades sociales y educativas, sobre todo en relación con las profesiones ofrecidas y el campo laboral de las mismas, promoviendo una capacitación integral con especial énfasis en la formación de valores. Además, deben apuntar a las transformaciones sociales para hacer posible el éxito del esfuerzo educativo (Zufiaurre \& Gabari, 2000). Esto implica considerar las demandas de la sociedad de la información y del conocimiento: incorporar las tecnologías de la información y la comunicación (TIC) en la enseñanza y el aprendizaje, apostar por la innovación y la creatividad y desarrollar investigación y pensamiento crítico.

\section{Problema de investigación y método}

Este estudio se enmarca en la problemática de la formación de profesores en Chile, analizada desde las percepciones de sus protagonistas, el profesorado del sistema escolar de los establecimientos educativos y los docentes universitarios encargados de la formación de educadores, alrededor de temas clave del proceso formativo y desempeño docente.

\section{Objetivo del estudio.}

Analizar las percepciones en torno a seis procesos fundamentales (evaluación, aprendizaje, innovación, investigación, perfeccionamiento y relación universidad/escuela/comunidad) en la formación de profesores desde la óptica de maestros del sistema educativo y de docentes universitarios formadores de profesores. 


\section{Diseño de investigación.}

El presente análisis se inscribe en un diseño investigativo no experimental de tipo transeccional descriptivo, es decir, tiene como propósito categorizar y proporcionar una visión de un fenómeno o situación mediante lo que demandan sus principales actores, docentes universitarios y del sistema escolar (Albert, 2007; Canales, 2006; Creswell, 2003).

\section{Sujetos informantes.}

Los sujetos informantes corresponden a una muestra no probabilística de 174 profesores chilenos de los sistemas escolar y universitario. 95 maestros laboran en el sistema escolar en una región del país, en establecimientos educativos de dependencia pública y semipública. Un $75 \%$ de los sujetos son mujeres y un $41 \%$ presenta más de veinte años de experiencia docente. 79 educadores trabajan en una universidad formadora de profesores, un 49,4\% son mujeres. El $46 \%$ de los profesores universitarios tiene una experiencia docente entre tres años y diez años, seguido de un $24 \%$ que tiene más de veinte años de experiencia.

Se está en presencia deunmuestreo subjetivo por decisión razonada, esto es, las unidades de la muestra no se eligen usando procedimientos probabilísticos, sino en función de algunas de sus características específicas (Corbetta, 2007).

\section{Instrumentos.}

Se elaboraron dos cuestionarios ad hoc para estudiar la percepción de los grupos. Para los maestros universitarios se diseñó un conjunto de once dimensiones que abarcaron 181 ítems con una escala tipo Likert de tres niveles de respuesta (en desacuerdo, ni de acuerdo ni en desacuerdo y de acuerdo). Para los profesores del sistema escolar, el cuestionario se confeccionó sobre un conjunto de trece dimensiones y 188 ítems, igualmente con una escala tipo Likert de tres niveles de respuesta.

\section{Procedimiento de análisis de los datos.}

El análisis estadístico de las respuestas de los profesores del sistema escolar y docentes universitarios, se realizó con el programa SPSS Statistics 22.0. Para fines de este estudio se seleccionaron seis dimensiones: evaluación, aprendizaje, innovación, investigación, perfeccionamiento y relación universidad/escuela/ comunidad, todas ellas en el contexto de la formación de profesores.

Cabe destacar que la encuesta aplicada no fue idéntica para ambos grupos, atendiendo a los objetivos que presentan la escuela y la universidad. Para aquellas preguntas idénticas, se introdujo un análisis por nivel educativo alcanzado.

\section{Presentación de los resultados}

\section{Análisis de la encuesta de opinión.}

\section{Datos sociodemográficos de los pro- fesores del sistema escolar.}

El grupo de profesores que respondió la encuesta de opinión estuvo conformado por 95 educadores del sistema escolar, de los cuales un $44 \%$ se desempeña en establecimientos públicos y un $56 \%$ trabaja en instalaciones de dependencia semipública. Un dato relevante es que el $59 \%$ de las mujeres y el $46 \%$ de los hombres labora en instituciones educativas con índice de vulnerabilidad escolar (IVE) ${ }^{2}$ muy alto (gráfica 1), esta es una señal de la condición socioeconómica de los estudiantes y sus familias. A pesar de lo anterior, un $81 \%$ de los encuestados declara no recibir un complemento monetario por desempeño difícil.

Respecto al nivel de estudios, se destaca que un $18 \%$ de los encuestados posee el grado académico de licenciado, un $41 \%$ el título 
profesional de profesor/educador de párvulos; un $3 \%$ cuenta con diplomado, un $16 \%$ tiene postítulo; y un $12 \%$ tiene una maestría.

\section{Gráfica 1.}

Profesores que trabajan en establecimientos educativos con índice de vulnerabilidad escolar muy alto

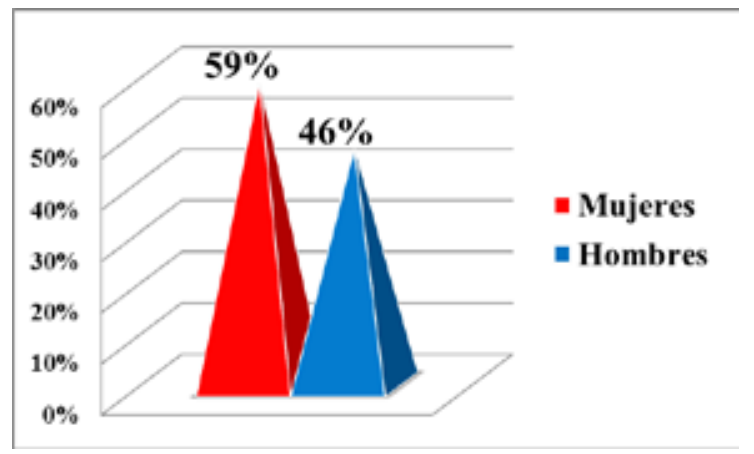

Fuente: elaboración propia.

\section{Docentes universitarios.}

La encuesta fue respondida por 79 profesores universitarios, de los cuales un 67 $\%$ declara tener una maestría (gráfica 2). Cabe destacar que un $47 \%$ tiene una experiencia docente en la universidad de entre tres años y diez años; y un $24 \%$ tiene una experiencia superior a veinte años. Tres de cada cuatro encuestados realiza sus clases en carreras de pregrado.

Gráfica 2.

Formación académica docentes universitarios

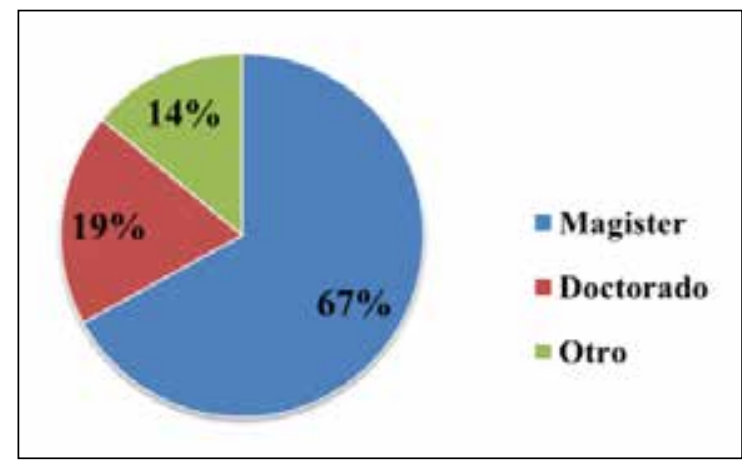

Fuente: elaboración propia.
A continuación se analizará cada una de las dimensiones abordadas en este estudio, desde la perspectiva de los profesores del sistema escolar y de los docentes universitarios formadores de profesores.

\section{El proceso de evaluación.}

Las prácticas evaluativas revelan una alta adhesión entre los encuestados, como lo manifiesta el $87 \%$ de los educadores del sistema escolar y el $78 \%$ de los maestros universitarios, quienes plantean que para mejorar el desempeño docente es imprescindible evaluar la práctica docente. Como declara Arbesú (2008), "la evaluación tiene que servir como un proceso de diálogo, comprensión y mejora de la práctica educativa $[\ldots]$ es el docente quien tiene que reflexionar sobre su propia práctica y decidir las transformaciones que realizará en su trabajo cotidiano" (p. 36).

En esta misma línea, nueve de cada diez profesores expresa que mejorar la evaluación impacta positivamente en la calidad de los procesos y resultados formativos. "La función formativa de la evaluación de la enseñanza, enfatiza la reflexión del profesor como una forma de generar conocimiento y analizar sistemáticamente distintos aspectos de los profesores al enseñar" (p. 291).

Sin embargo, la incorporación de la autoevaluación y coevaluación como práctica habitual del profesorado escolar, alcanza menor nivel de adhesión (54\%). El tiempo es una variable que influye en las posibilidades de participar en la evaluación: solo un $35 \%$ de los docentes universitarios considera que dispone de tiempo para la autoevaluación, mientras el $41 \%$ estima que la escasa disponibilidad de tiempo es una limitante para cumplir con un sistema de evaluación riguroso.

Una multiplicidad de factores determina que la evaluación sea un proceso complejo. En 
los profesores del sistema escolar, no genera una tendencia mayoritaria la aseveración "en las condiciones actuales es casi imposible utilizar los resultados de la evaluación para la reorientación del proceso educativo". Un 37 \% no está de acuerdo ni en desacuerdo con esta frase, un $37 \%$ está en desacuerdo, mientras un $27 \%$ está de acuerdo. Aunque casi el $90 \%$ de los profesores del sistema escolar juzga a la evaluación como una oportunidad para mejorar el desempeño docente, persiste la idea, en un $39 \%$ de ellos, de que la finalidad primordial de la evaluación es calificar el aprendizaje de los estudiantes (gráfica 3).

Gráfica 3.

El proceso de evaluación

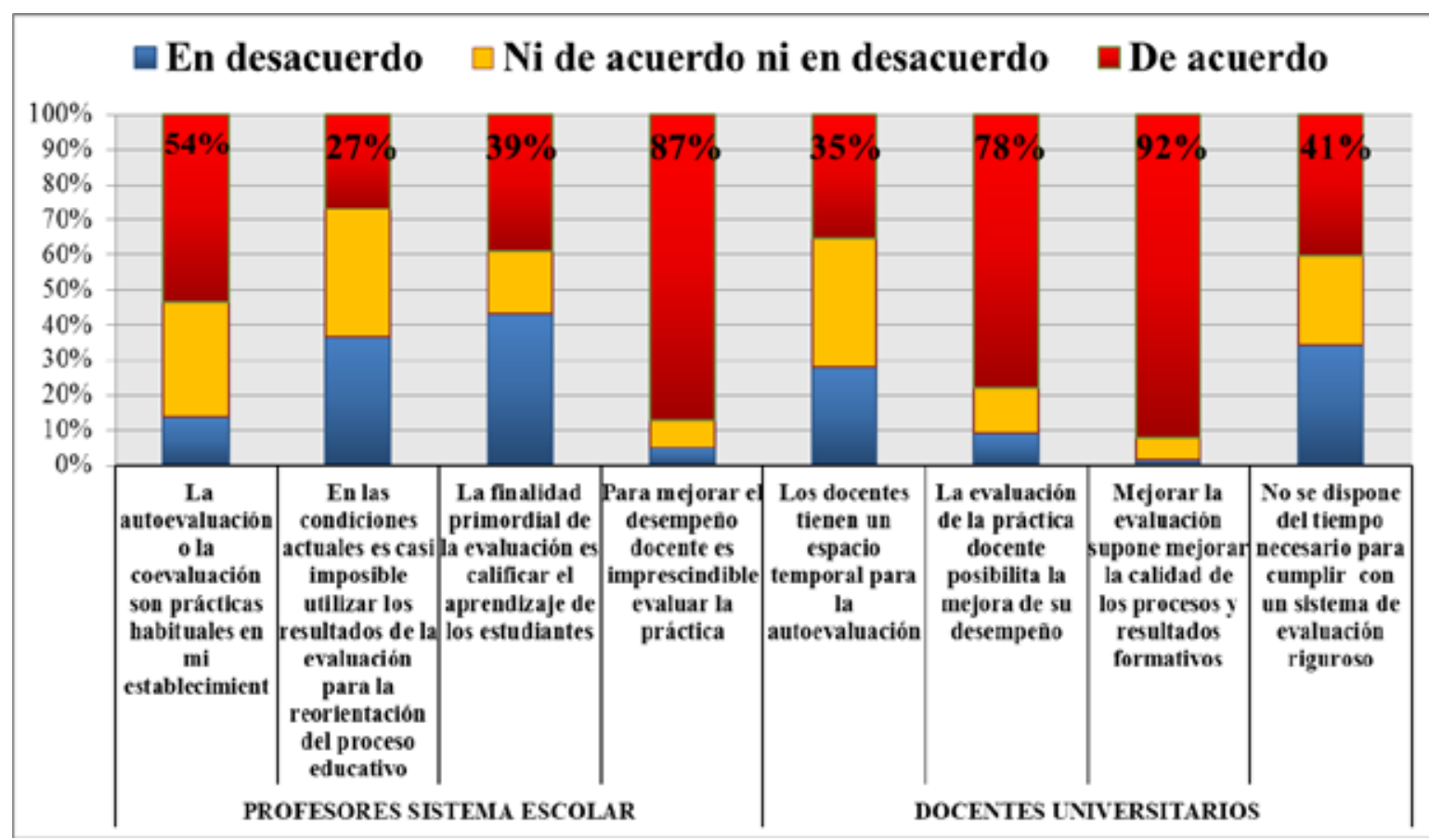

Fuente: elaboración propia.

\section{El proceso de aprendizaje.}

Los ítems que se consultaron a profesores del sistema escolar y docentes universitarios son idénticos, cuyos resultados se detallan a continuación. El $34 \%$ de los educadores del sistema escolar y el $42 \%$ de los docentes universitarios considera que el aprendizaje es una actividad individual. En correspondencia con ese dato, uno de cada tres profesores sostiene que la actividad grupal hace invisible el trabajo individual. Cuando se introduce un análisis por nivel educativo, se aprecia que un $33 \%$ de maestros con título de profesor/educador de párvulos, se manifiesta de acuerdo con esta frase, mientras que un 34 $\%$ de los docentes con maestría está a favor de este enunciado. 
Gráfica 4.

Aprendizaje: respuestas en el nivel "de acuerdo"

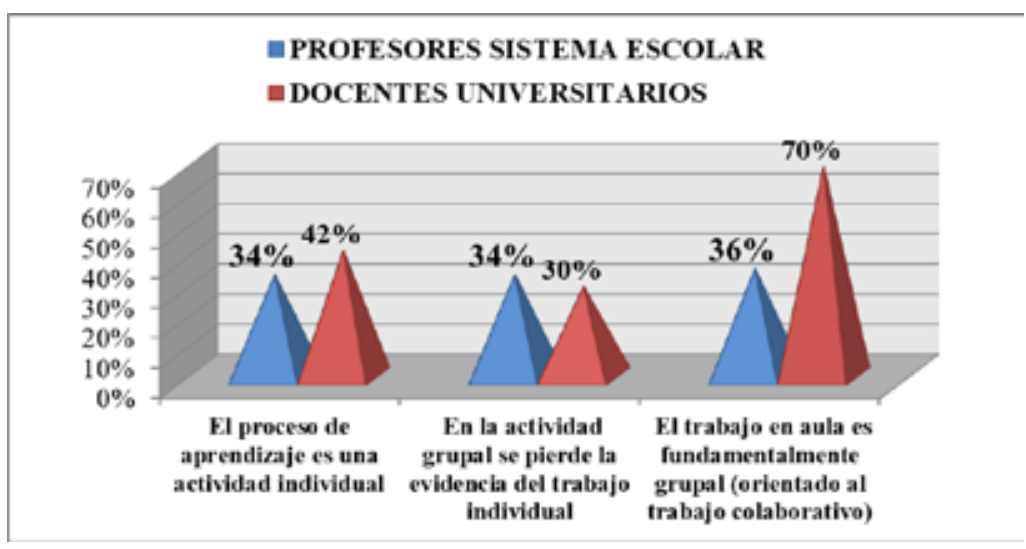

Fuente: elaboración propia.

La implementación de tareas colaborativas genera las siguientes percepciones: un 45 $\%$ de los profesores del sistema escolar con título de profesor/educador de párvulos estima que el trabajo en aula es fundamentalmente grupal, mientras que un $70 \%$ de los docentes universitarios orienta su labor en el aula hacia actividades de aprendizaje colaborativo y cooperativo (gráfica 4). Son muchos los autores (Felder \& Brent, 2007) que han realizado investigaciones sobre los beneficios del trabajo colaborativo en diversas disciplinas, y en particular, en el desarrollo de habilidades para resolver problemas, trabajar en equipo, lograr un mejor entendimiento conceptual y promover el pensamiento crítico.

Las actividades colaborativas las impulsan nueve de cada diez maestros del sistema escolar; no obstante, un $37 \%$ de ellos considera que es difícil su implementación. Por otro lado, un $53 \%$ plantea que el aprendizaje efectivo se desarrolla en un ambiente ordenado y silencioso (gráfica 5). Entre las diversas razones que condicionan su promoción en el ámbito escolar, se encuentran el insuficiente conocimiento de esta estrategia y el temor de los docentes a perder el control de la disciplina y no cubrir los contenidos del currículo.

Gráfica 5.

Aprendizaje y trabajo colaborativo: profesores del sistema escolar

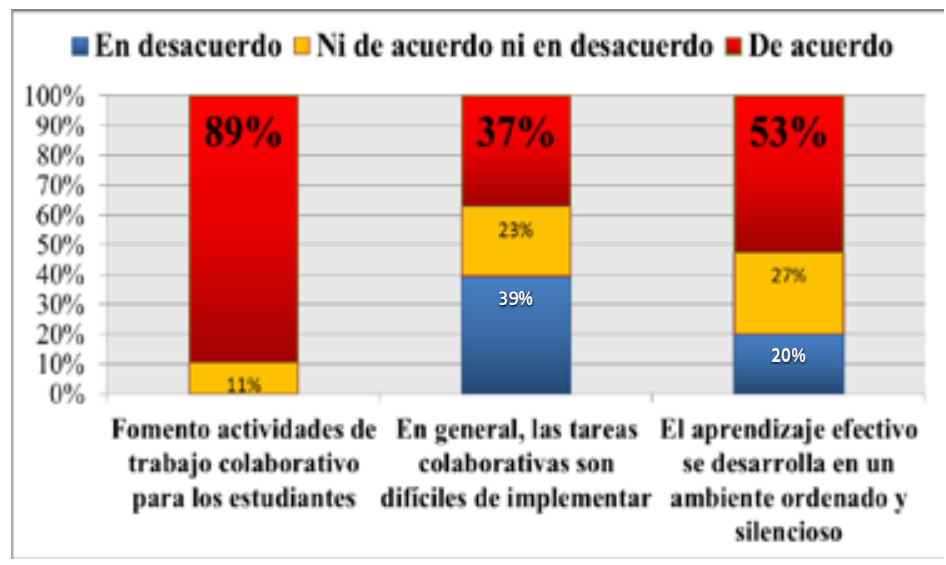

Fuente: elaboración propia. 


\section{El proceso de innovación.}

En el caso de los profesores del sistema escolar, las preguntas acerca de la innovación se dirigen a medir su promoción en el establecimiento escolar, frecuencia y relación con la investigación. En cambio, las preguntas para docentes universitarios consultan sobre la ejecución de innovaciones, la disponibilidad de recursos tecnológicos y la creencia sobre cultura innovadora.

La innovación es valorada en el ejercicio profesional de los profesores del sistema escolar: un $44 \%$ de los docentes del sistema escolar está de acuerdo con la afirmación: "las prácticas educativas innovadoras son habituales en su establecimiento", mientras que un $63 \%$ declara promover estas iniciativas.
En el contexto universitario, cuatro de cada cinco docentes emprende prácticas educativas innovadoras, para lo cual cuentan con recursos tecnológicos (75\%). En esta línea, según un $63 \%$ de los docentes universitarios la metodología didáctica tradicional es uno de los factores que obstaculiza el desarrollo de una cultura innovadora al interior de las instituciones educativas. En resumen, las iniciativas innovadoras son promovidas por los dos grupos de informantes.

Un dato que requiere análisis posteriores, se refiere a que un $35 \%$ de los educadores del sistema escolar declara que estas iniciativas surgen de los resultados aportados por sus propias investigaciones (gráfica 6), variable que se analizará enseguida.

Gráfica 6.

El proceso de innovación

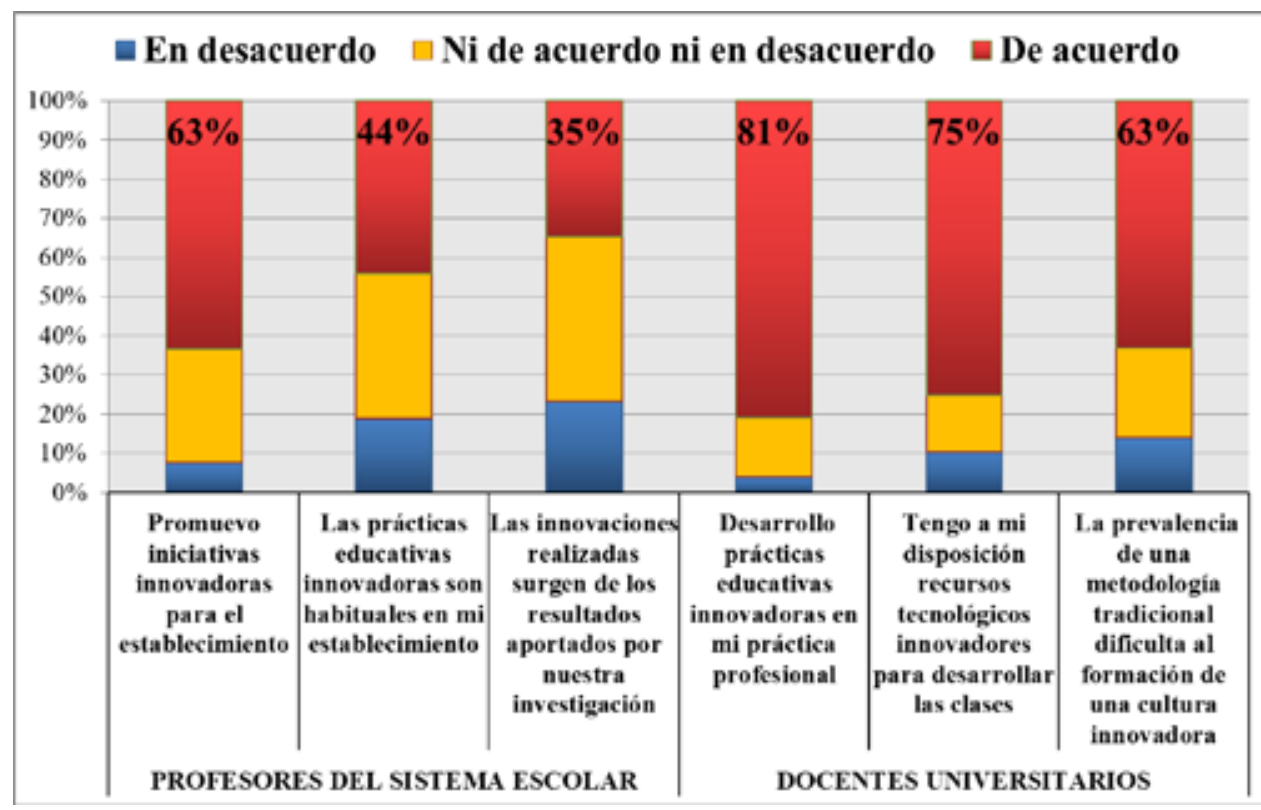

Fuente: elaboración propia.

La integración entre la docencia y la investigación para promover la innovación, ayuda a que los docentes se conviertan en los gestores del cambio educativo. Rodríguez (2000) plantea que los maestros son por definición los protagonistas de la transformación educativa; sin su compromiso este acontecimiento no es viable. Los maestros están detrás de los principales factores que dinamizan el cambio. Los actores de las prácticas pedagógicas innovadoras, con sus experiencias y reflexiones, son quienes propician la aparición de nuevas teorías pedagógicas. 


\section{El proceso de investigación.}

Los interrogantes de esta sección son diferentes para cada grupo. Para los profesores del sistema escolar se formularon preguntas que intentaban identificar la existencia de la práctica investigativa, teniendo como antecedente que la investigación no es liderada frecuentemente por las propias escuelas. En el contexto universitario, los ítems se orientan a consultar por la forma en que la investigación se relaciona con la praxis educativa, las teóricas pedagógicas y la actualización docente.

Un $28 \%$ de los profesores del sistema escolar sostiene que en sus escuelas se realiza investigación educativa y, acorde con esto, que existe una cultura de investigación educativa. Sin embargo, es interesante notar que se incrementa el porcentaje de profesores a un $40 \%$ que manifiesta la existencia de una indagación sistemática sobre sus problemas en los establecimientos escolares donde trabajan (gráfica 7); empero, un $38 \%$ afirma que no está ni de acuerdo, ni en desacuerdo con esto.

No obstante, no existe igual número de maestros que asocia las problemáticas educativas como sustento para la investigación y como generadoras de conocimiento para incrementar el cuerpo teórico del saber pedagógico. ¿Qué factores podrían estar condicionando esta situación?, Rodríguez y Castañeda (2001) señalan que si bien existe un amplio acuerdo acerca de la importancia de la investigación educativa y la necesidad de formar a los profesores en procesos de innovación e investigación, no parece que haya consenso respecto al significado del conocimiento generado por los docentes para la construcción de teorías (p. 113).

Es por eso que las investigaciones hechas por los docentes en el contexto escolar, no tienen igual significación para todos, así desde la perspectiva de quien produce el conocimiento, este solo tiene validez práctica; mientras otros consideran que el saber que producen los profesores debe enriquecer no solo la práctica, sino también la teoría educativa.

En cuanto a la investigación realizada por docentes universitarios, un $43 \%$ manifiesta que "en nuestra facultad la investigación es fuente de y para la teoría pedagógica"; además, para uno de cada dos encuestados las mejoras docentes tienen como sustento la investigación-acción o la investigación aplicada (gráfica 7). En armonía con Pérez-Gómez (1995) "el conocimiento pedagógico no será útil ni relevante a menos que se incorpore al pensamiento y acción de los agentes [...] así pues, la intencionalidad y sentido de toda investigación educativa es la transformación y mejoramiento de la práctica" (p. 117).

Gráfica 7.

El proceso de investigación

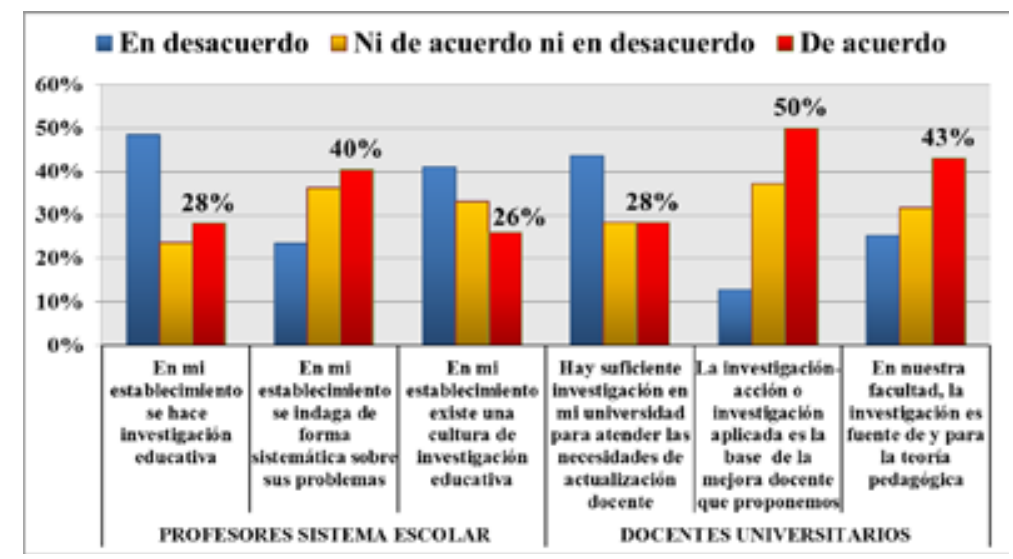

Fuente: elaboración propia. 
Respecto a la dedicación a actividades de investigación, un $57 \%$ de los docentes universitarios indica no ejercerla de manera habitual. El nivel educativo es relevante en este caso: un $39 \%$ de los educadores con maestría declara realizar investigación, mientras que esta cifra asciende al $73 \%$ en aquellos profesores que poseen un doctorado.

Del $43 \%$ de los docentes que sí investiga, un $77 \%$ invierte menos de seis horas semanales y solo el $6 \%$ de los encuestados destina más de 24 horas semanales a esta actividad (gráfica 8). Los datos revelan que la importancia que se atribuye a la investigación como impulsora de las mejoras docentes, no se implementa en la práctica con una fuerte dedicación de los docentes. De este modo, los docentes universitarios cumplen principalmente un rol de profesores y no de investigadores.

Gráfica 8.

Docentes universitarios que habitualmente ejercen investigación

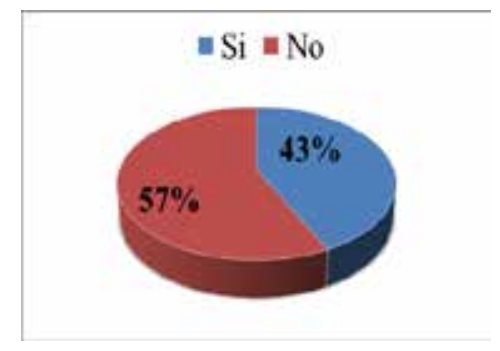

Fuente: elaboración propia.

\section{El proceso de perfeccionamiento docente.}

En este ítem las preguntas que se propusieron a profesores del sistema escolar y docentes universitarios son idénticas. Los resultados muestran que los programas de perfeccionamiento y de posgrado, como instancias de formación continua, son ampliamente valorados: más del $80 \%$ de los profesores del sistema escolar y de los docentes universitarios considera estas instancias de capacitación como la oportunidad de fortalecer sus competencias docentes e impactar positivamente en las prácticas pedagógicas (gráfica 9). Donoso (2008) se refiere al perfeccionamiento docente en los siguientes términos:

Conceptualmente, el perfeccionamiento docente incluye tanto la formación inicial de profesores como la actualización profesional. Dado que son herramientas políticas diferentes que responden a objetivos alternativos, requieren análisis diferenciados. En Chile son vistos como dos aspectos complementarios aunque propios. La formación inicial corresponde al diseño de la profesión en su sentido amplio, busca desplegar las competencias para el desempeño de la profesión. El perfeccionamiento -tradicionalmente- ha sido establecido para alcanzar un impacto más próximo y está remitido a competencias específicas, actualizaciones o desarrollo de nuevas competencias profesionales (p. 438).

Por el contrario, cuando se consulta sobre la calidad de los programas, disminuye el nivel de acuerdo. No más de un $49 \%$ de los profesores de establecimientos escolares cree que la universidad imparte programas de perfeccionamiento de calidad, cifra que en el caso de los docentes universitarios alcanza el $73 \%$. Un análisis por nivel educativo alcanzado, revela que un $45 \%$ de profesores con título de profesor/educador de párvulos, está de acuerdo con la afirmación, mientras que un $76 \%$ de los docentes con maestría tiene esta percepción. Estas diferencias requieren de análisis más detallados, puesto que si bien la formación continua se valora como una oportunidad, la calidad de estos programas presenta percepciones dispares.

Un $56 \%$ de los docentes del sistema escolar está de acuerdo con la aseveración "los programas de perfeccionamiento son elegidos por la institución con base en un diagnóstico de necesidades". La afirmación se plantea del siguiente modo para los docentes universitarios: "los programas de perfeccionamiento se diseñan con base en el diagnóstico de necesidades institucionales", lo que alcanza un $63 \%$ 
de acuerdo. Según el nivel educativo logrado, un $62 \%$ de profesores con título de profesor/ educador de párvulos, se encuentra de acuerdo, mientras que el mismo porcentaje de los docentes universitarios con maestría se manifiesta en esta línea.

En cuanto al rol que asume el Ministerio de Educación como facilitador de los procesos de perfeccionamiento continuo de los docentes, el $49 \%$ y el $51 \%$ de los profesores de establecimientos educativos y docentes universitarios respectivamente, están de acuerdo con esta aserción. En otras palabras, la percepción está polarizada respecto a considerar a esta institución como garante de la formación continua. Como apunta Donoso (2008):

En grandes cifras el perfeccionamiento docente sigue siendo un área desregulada del sistema, evidenciando con ello el problema de institucionalidad que afecta al sistema educa- cional chileno y, en especial, al Ministerio de Educación como garante social de la calidad educacional (p. 13).

Cuando se introduce el nivel educativo, los datos muestran que un $46 \%$ de los profesores escolares con título de profesor/educador de párvulos juzga que el Ministerio de Educación es facilitador del perfeccionamiento docente; esta percepción la manifiesta el $52 \%$ de los maestros universitarios con maestría.

La aseveración "los programas de posgrado son una instancia de fortalecimiento de las competencias docentes" alcanza resultados muy similares por nivel educativo: un $79 \%$ de los educadores con maestría se declara de acuerdo, igual que un $84 \%$ de los profesores con título de profesor/educador de párvulos. Se constituye en un tema pendiente indagar otros ámbitos que pueden afianzarse a través de programas de posgrado.

Gráfica 9.

Perfeccionamiento docente: respuestas en el nivel "de acuerdo"

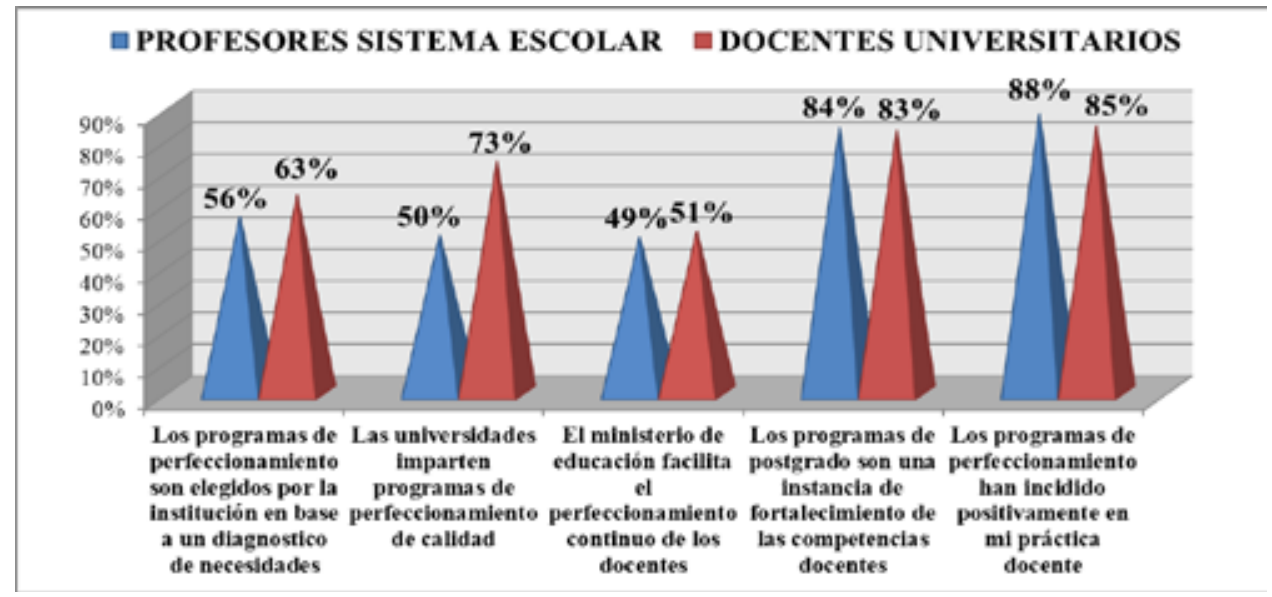

Fuente: elaboración propia.

Cuando se consulta en concreto por la disponibilidad de tiempo para participar en cursos de perfeccionamiento de manera regular, solo un tercio de los docentes universitarios señala que puede asistir. Así mismo, la falta de tiempo se transforma en un obstáculo para el perfeccionamiento docente en opinión del $71 \%$ de los profesores del sistema escolar (gráfica 10). Este resultado es relevante en la medida que los profesores requieren de conocimientos actualizados para mejorar su práctica docente. 
Gráfica 10.

Perfeccionamiento y tiempo disponible

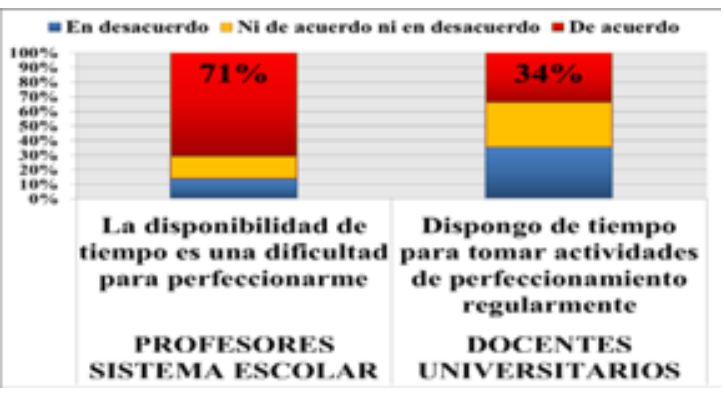

Fuente: elaboración propia.
Se consultó a los docentes universitarios su percepción sobre la formación basada en competencias, en relación con su viabilidad y requerimientos. Los resultados arrojan que a pesar de que en Chile la formación con soporte en competencias no es un tema reciente, aún persisten las dificultades y resistencias para su implementación; en efecto, un bajo porcentaje de docentes la perciben como viable ( $22 \%)$, ya que exige una revisión de la metodología didáctica para su aplicación (84 \%) (gráfica 11).

Gráfica 11.

Formación basada en competencias: respuestas en el nivel "de acuerdo"

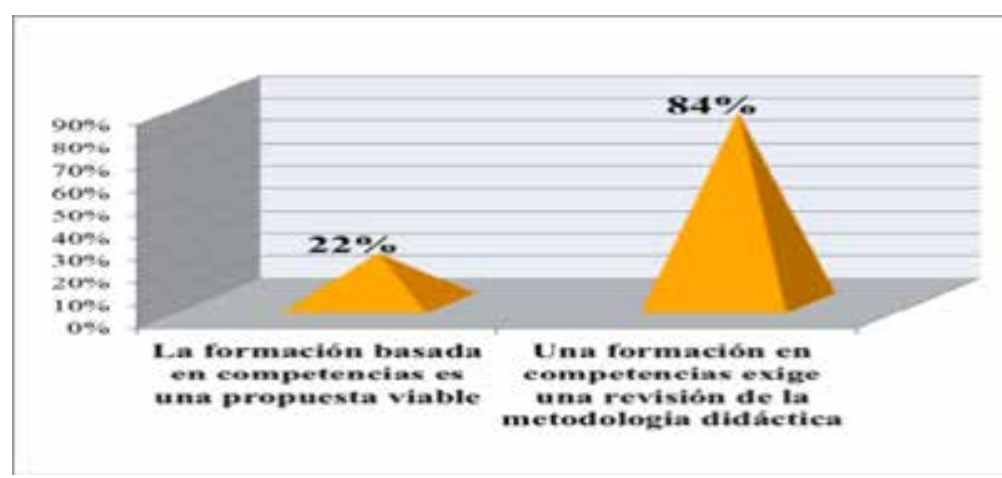

Fuente: elaboración propia.

Las apreciaciones de los docentes coinciden con algunas de las conclusiones emanadas de la investigación de García y Castro (2012):

La formación permanente basada en competencias se erige como medio para ofrecer una respuesta adecuada a una realidad educativa, en continua transformación, que vivencia el profesorado en las aulas [...] Sin embargo se presentan varias dificultades para su implementación. Entre ellas, llama la atención el bajo nivel de competencia profesional autopercibida por parte del profesorado: los docentes no se sienten bien preparados a pesar de servirse de acciones formativas. Un punto débil encontrado en la valoración de estas acciones formativas es la descontextualización de las mismas y la necesidad de realizar adaptaciones (pp. 319-320).

\section{Relación del establecimiento educa- tivo con la universidad y la comuni- dad escolar.}

Los ítems que se incluyen en esta sección son idénticos para profesores del sistema escolar y para docentes universitarios. El análisis de los resultados permite indicar que ambos actores presentan respuestas similares. La afirmación: "los vínculos con universidades se orientan explícitamente a la mejora de la acción docente" alcanza respuestas en la categoría de acuerdo en un $47 \%$ de profesores del escolar, y $51 \%$ de docentes universitarios. Según el nivel educativo obtenido, un $56 \%$ de los profesores con título de profesor/educador de párvulos, está de acuerdo con la aseveración, mientras que un $50 \%$ de los docentes universitarios con maestría se manifiesta en la misma línea. 
Un $51 \%$ de profesores del sistema escolar y un $46 \%$ de los docentes universitarios considera que "las relaciones con la comunidad del sector de ubicación del establecimiento son fluidas y beneficiosas". A la luz del nivel educativo adquirido, un $67 \%$ de los profesores del sistema escolar con título de profesor/ educador de párvulos se encuentra de acuerdo con esta frase; mientras que un $33 \%$ de los docentes con título de maestría se expresa en este sentido. A diferencia de otros ítems, este aspecto da cuenta de percepciones desiguales en las cuales se podría indagar, tanto para fortalecer el rol de las escuelas como para iniciar un proceso de vinculación de las universidades con el medio.

Por otro lado, un $29 \%$ de los profesores de instituciones escolares, considera que "la universidad promueve la comunicación entre el profesorado colaborador más allá de sus funcio- nes en el establecimiento o de la relación que pudiera darse con un establecimiento escolar". Un mayor porcentaje de docentes universitarios (47\%), está de acuerdo con esta afirmación (gráfica 12). Según nivel educativo, esta percepción se presenta en el $39 \%$ de los maestros con título de profesor/educador de párvulos, mientras que un $32 \%$ de los docentes universitarios con maestría opina en este sentido.

Otra pregunta da cuenta de esta percepción: para un $43 \%$ de los educadores del sistema escolar "las relaciones con la universidad fortalecen los diversos ámbitos del proyecto educativo". Por nivel educativo alcanzado, un $53 \%$ de los profesores con título de profesor/educador de párvulos se manifiesta de acuerdo. Estos resultados revelan que, en criterio de los profesores, los vínculos entre las universidades y el sistema escolar aún no se hallan suficientemente consolidados.

Gráfica 12.

Relación universidad/escuela/comunidad: respuestas en el nivel "de acuerdo"

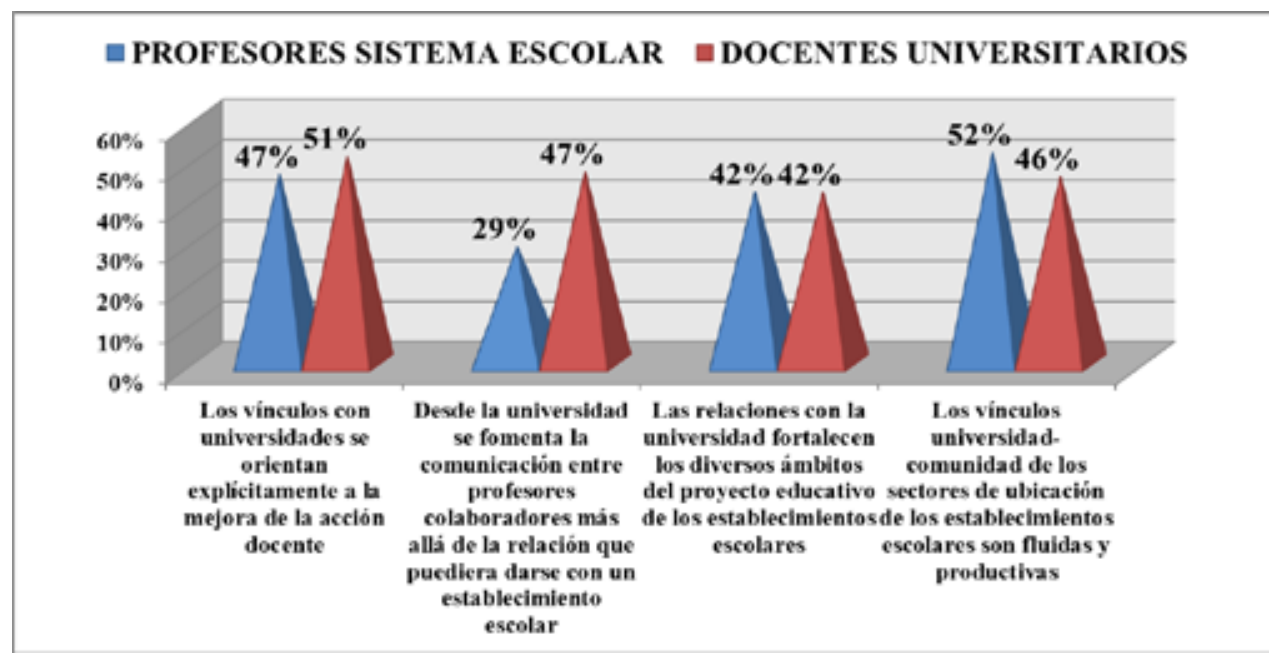

Fuente: elaboración propia.

\section{Conclusiones}

Las propuestas de cambio que demanda la formación del profesorado deben integrar los resultados de las investigaciones e inno- vaciones educativas que se realizan en los contextos educativo y social. Los docentes pueden elaborar teoría pedagógica a partir de la inves- 
tigación educativa, eliminando la disociación que tradicionalmente se ha planteado entre teoría y práctica, que deja la primera a los investigadores y la segunda a los profesores cuando se enfrentan a las tareas cotidianas de su actuar docente (Rodríguez \& Castañeda, 2001).

Desde esta óptica, las opiniones vertidas por los docentes se aproximan al conocimiento de sus teorías y percepciones relacionadas con los diversos ámbitos que implica su acción pedagógica, ya sea desde la investigación, innovación, aprendizaje, perfeccionamiento y evaluación.

Se destaca la necesidad de formar a los futuros educadores con un adecuado equilibrio entre la formación pedagógica, incorporando en ello los conocimientos necesarios de las ciencias más directamente vinculadas a la enseñanza y el aprendizaje, y a la dimensión disciplinaria de acuerdo con el nivel del sistema educativo y las áreas del conocimiento donde se desempeñarán. El profesor debe saber "lo que enseña" y "cómo lo enseña".

La investigación en el ámbito escolar presenta dos resultados que pueden constituir una fuente para próximos análisis: de acuerdo con el $40 \%$ de profesores encuestados se realiza indagación sistemática en la escuela donde trabajan; mientras que el $35 \%$ declara que las iniciativas innovadoras surgen de los resultados aportados por sus propias investigaciones. Estos datos pueden configurar una posibilidad de generar espacios de investigación-acción, donde los maestros escolares puedan vincularse con docentes universitarios para promover el mejoramiento de las prácticas pedagógicas desde la experiencia en los establecimientos escolares.

La investigación en el ámbito universitario es menos frecuente de lo esperado: menos de la mitad de los formadores de profesores encuestados realiza investigación. La falta de tiempo es uno de los principales impedimentos para conformar equipos de trabajo interdiscipli- nario. Estos docentes tampoco declaran investigar sistemáticamente sobre su propio quehacer. De este modo, la investigación en las instituciones universitarias no contribuye como debiera al enriquecimiento de la deficitaria producción de conocimiento sobre educación en el país, a fundamentar con evidencias el mejoramiento de la formación, ni a crear una cultura investigativa en los estudiantes de pedagogía.

En cuanto a la vinculación de la universidad, los programas de formación de profesores del alma máter deberían desarrollar un plan de difusión de las actividades relacionadas con la responsabilidad social universitaria, que fortalezca el nexo entre la universidad y la comunidad y visibilice el compromiso social de esta con la comunidad, la región y el país. En esta misma línea, los programas de pedagogía evidencian la necesidad de potenciar la difusión de las carreras, la ausencia de oferta de educación continua y la falta de seguimiento de los egresados. Estas tres necesidades dan cuenta de la importancia de que el proceso formativo universitario no finalice en la universidad, sino que trascienda a la comunidad y promueva el aprendizaje continuo y a lo largo de la vida de los docentes.

Reafirmando los aspectos analizados, un $85 \%$ de los profesores del sistema escolar considera que "las universidades deben colaborar con los establecimientos para investigar las necesidades de formación de los docentes". La investigación y la vinculación universidadestablecimiento escolar son aspectos clave para mejorar la formación de profesores, en un escenario que demanda cada vez con más fuerza el trabajo conjunto entre ambas instancias educativas. Así mismo, un $71 \%$ de los docentes universitarios estima que "las problemáticas de la comunidad, en sentido general, son objeto de insumo en los procesos de formación de los profesionales de la enseñanza", lo que da cuenta de que distintos elementos del contexto sociocultural se entienden como parte de la formación de profesores. 
En relación con el proceso de aprendizaje, en particular mediante trabajo colaborativo, urge comprender por qué, a pesar de la existencia de evidencia teórica y práctica sobre sus beneficios, este se promueve con dificultades en las escuelas. Trabajar de manera cooperativa es algo complejo que requiere de tiempo. Un primer paso ineludible es fomentar una cultura de la cooperación en la escuela, que involucre a toda la comunidad educativa para comprender y abordar este problema.

En armonía con García y Castro (2012) uno de los criterios que definen la calidad de la educación es la formación permanente del profesorado. No cabe duda de que la formación permanente del cuerpo docente se enfrenta a nuevos desafíos que requieren un esfuerzo por innovar, buscar soluciones, adaptar la formación a diversos y cambiantes contextos y reforzar la coordinación entre la formación inicial y la formación permanente, en función del mejoramiento del desempeño profesional docente. El desarrollo de las competencias en el alumnado, se ha constituido en uno de los elementos novedosos en el ámbito educativo que demanda al profesorado un cambio en su papel docente, dirigido a una formación permanente basada en competencias.

En este panorama se justifica la necesidad de escuchar, recoger y valorar la voz del profesorado para conocer las fortalezas y limitaciones de los planes de formación y poder mejorar su competencia profesional, que sin duda, continúa siendo un desafío para todos.

\section{Referencias}

Albert, M. (2007). La investigación educativa. Madrid: Claves teóricas.

Arbesú, M. I. (2008). Evaluación formativa de la docencia universitaria. En CINDA (Ed.), Evaluación del desempeño docente y calidad de la docencia universitaria (pp. 290-291). Santiago, Chile: Alfabeta Artes Gráficas.
Canales, M. (2006). Metodologías de investigación social. Introducción a los oficios. Santiago de Chile: LOM.

Cárdenas, A., Rodríguez, A., \& Torres, R. (2000). El maestro protagonista del cambio educativo. Bogotá: Magisterio.

Centro Interuniversitario de Desarrollo-[CINDA]. (2009). Diseño curricular basado en competencias y aseguramiento de la calidad en la educación superior. Santiago de Chile: Alfabeta Artes Gráficas.

Cisternas, T. (2013). Contextos, contingencia e intereses en el proceso de investigar la formación docente: Un estudio de caso. Revista Calidad de la Educación, 38, 116-117.

Corbetta, P. (2007). Metodología y técnicas de investigación social. Madrid: McGraw Hill.

Creswell, J. (2003). Research design. Qualitative, quantitative and mixed methods and approaches. Londres: Sage Publications.

Donoso, D. S. (2008). El perfeccionamiento docente en Chile (1990-2007): ¿Estado versus mercado?. Revista Brasileira de Educação, 13(39), 437-593. Recuperado de http://www.scielo.br/pdf/rbedu/ v13n39/03.pdf

Felder, R., \& Brent, R. (2007). Cooperative Learning. En P. A. Mabrouk (Ed.), Active Learning: Models from the Analytical Sciences (pp. 34-53). Washington: American Chemical Society.

Frabboni, F. (2001). El libro de la pedagogía y la didáctica I: la educación. Madrid: Editorial popular.

García, R., \& Castro. Z, A. (2012). La formación permanente del profesorado basa- 
da en competencias. Estudio exploratorio de la percepción del profesorado de Educación Infantil y Primaria. Educatio Siglo XXI, 30(1), 297-322. Recuperado de http://revistas.um.es/educatio/article/ view/149251

Gonzáles S., Río, E., \& Rosales, S. (2001). El Currículum oculto en la escuela. Buenos Aires: Lumen Humanitas.

Hashweh, M. (2005). Teacher pedagogical constructions: a reconfiguration of pedagogical content knowledge. Teachers and Thinking: Theory and Practice, 11, 273292.

Joyce, B., Marsha, W., \& Calhoun, E. (2002). Modelos de enseñanza. Barcelona: Gedisa.

Kansanen, P., Tirri, K., Meri, M., Krokfors, L., Husu, J., \& Jyrhämä, R. (2000). Teachers' pedagogical thinking. Neva York: American University Studies.

Libedinsky, M. (2001). La innovación en la enseñanza. Buenos Aires: Paidós.

Médina, A., \& Salvador, F. (2002). Didáctica general. Madrid: Prentice Hall.

Menin, O. (2001). Pedagogía y Universidad. Currículo, didáctica y evaluación. Santa Fe: Ediciones Homosapiens.

Ministerio de Educación Gobierno de Chile -[MINEDUC]. (2013). Marco para la Buena Enseñanza. Recuperado de http:// ww2.educarchile.cl/UserFiles/P0001/ File/Marco\%20para\%20Buena\%20 Ense\%C3\%B1anza.pdf

Monereo, C., Castillo, M., Clariana, M., Palma, M., \& Pérez, M. (2004). Estrategias de enseñanza y aprendizaje. Formación del profesorado y aplicación en la escuela. Barcelona: Graó.
Muchmore, J. (2004). A teachers' life. San Francisco: Backalong books.

Neary, M. (2002). Curriculum studies in post-compulsory and adult education. A teacher's and student teacher's study guide. Cardiff: Nelson Thornes.

Organización para la Cooperación y el Desarrollo Económicos [OCDE]. (2004). Revisión de políticas nacionales en educación. Santiago de Chile: OCDE; MINEDUC.

Organización para la Cooperación y el Desarrollo Económicos [OCDE]. (2013). Synergism for better learning: An international perspective on evaluation and assessment in Education. Paris: Autor.

Organicación de las Naciones Unidades para la Educación, la Ciencia y la Cultura. [UNESCO]. (1998, Octubre). La educación superior en el siglo XXI: visión y acción. Conferencia mundial sobre la educación superior. Paris.

Pérez-Gómez, A. (1995). Comprender la enseñanza en la escuela. Modelos metodológicos de investigación educativa. En J. Gimeno, \& A. Pérez-Gómez (Eds.), Comprender y transformar la enseñanza (pp. 115-136). Madrid: Morata.

Roberts, B. (2002). Biographical research. Philadelphia: Open University Press.

Rodríguez, A. (2000). Cambio y reformas en educación: el papel de los maestros. En A. Cárdenas, R. Torres, \& A. Rodríguez (Eds.), El maestro, protagonista del cambio educativo (pp. 79-159). Bogotá: Convenio Andrés Bello, Cooperativa Editorial Magisterio.

Rodríguez, J., \& Castañeda, E. (2001). Los profesores en contextos de investigación e 
innovación. Revista Iberoamericana de Educación, 25, 103-144. Recuperado de http://www.rieoei.org/rie25f.htm

Sandín, M. P. (2003). Investigación cualitativa en educación. Fundamentos y tradiciones. Madrid: Mc Graw Hill.

Sanjurjo, L. (2002). La formación práctica de los docentes. Reflexión y acción en el aula. Santa Fe: Homosapiens.

Scharle, A., \& Szabó, A. (2000). Learner autonomy. A guide to developing learner responsibility. Cambridge: Cambridge University Press.

Solar, M. I. (Mayo, 2013). Innovaciones en la formación de profesionales de la educación en Chile. Ponencia presentada en el 33rd Annual Seminar of the International Society for Teacher Education, Hong Kong.

Torre De la, S. (2004). Aprender de los errores. El tratamiento didáctico de los errores como estrategia de innovación. Buenos Aires: Magisterio.

Zepeda, S. (2007). Estudio sobre la percepción de la relación profesor-alumno entre estudiantes de colegios vulnerables de la región metropolitana. Revista Iberoanteramericana de Educación. 5(43), 1-13. Recuperado de http://www.rieoei.org/ deloslectores/1720Aguirre.pdf.

Zufiaurre, B., \& Gabari, M. (2000). Didáctica para maestras. Madrid: Editorial CCS. 
